\title{
Prognostic Value of Neutrophil-to-Lymphocyte Ratio in Obstructing Colorectal Cancer Treated by Endoscopic Stenting as a Bridge to Surgery
}

\author{
Jiwei Guo ${ }^{1}$, Aik Yong Chok ${ }^{1}$, Hui Jun Lim ${ }^{1}$ Wei Xuan Tay², Weng Kit Lye ${ }^{3}$, Lasitha Bhagya Samarakoon ${ }^{1}$, \\ Emile John Tan ${ }^{1}$, Ronnie Mathew ${ }^{1}$ \\ ${ }^{1}$ Department of Colorectal Surgery, Singapore General Hospital; ${ }^{2}$ Yong Loo Lin School of Medicine, National University of Singapore; ${ }^{3}$ Center \\ for Quantitative Medicine, Duke-NUS Graduate Medical School, Singapore, Singapore
}

Purpose: Neutrophil-to-lymphocyte ratio (NLR) has been reported to predict adverse survival outcomes among patients with colorectal cancer (CRC). This study evaluates the prognostic value of NLR among patients with obstructing CRC who successfully underwent stenting before curative surgery.

Methods: We retrospectively reviewed patients who underwent stenting before surgery. Patient demographics, tumor characteristics, perioperative outcomes, recurrence-free survival (RFS), and overall survival (OS) were analyzed. NLR was calculated from the differential white blood cell counts at least 4 days after successful stenting, before elective surgery. Optimal cutoff to dichotomize NLR was obtained by maximizing log-rank test statistic with recursive partitioning of KaplanMeier RFS and OS curves. The optimal cutoff for high NLR was $\geq 5$ at presentation before stenting, and $\geq 4$ after stenting. Results: Fifty-seven patients with localized obstructing CRC underwent successful endoscopic stenting before curative surgery. High NLR was associated with lymphovascular invasion $(\mathrm{P}=0.006)$ and apical lymph node involvement $(\mathrm{P}=0.034)$. Major perioperative complication(s) (hazard ratio [HR], 11.34; 95\% confidence interval [CI], 2.49 to 51.56; $\mathrm{P}<0.01$ ) and high NLR (HR, 3.69; 95\% CI, 1.46 to 9.35; P <0.01) negatively impacted OS on univariate and multivariate analyses. High NLR negatively impacted RFS on univariate analysis (HR, 2.91; 95\% CI, 1.29 to $6.60 ; \mathrm{P}=0.01$ ).

Conclusion: NLR of $\geq 4$ after stenting is an independent prognostic factor among patients with obstructing localized CRC who are successfully decompressed by endoscopic stenting before curative surgery.

\section{Keywords: Obstructing colorectal cancer; Neutrophil-to-lymphocyte ratio; Stents}

\section{INTRODUCTION}

Neutrophil-to-lymphocyte ratio (NLR) has been implicated with adverse oncological outcomes among colorectal cancer (CRC) patients, associated with worse overall survival (OS) and recur-

Received: Feb 20, 2020 - Revised: May 1, 2020 - Accepted: May 25, 2020 Correspondence to: Aik Yong Chok, FRCS

Department of Colorectal Surgery, Singapore General Hospital, Academia, 20 College Road, Singapore 169856, Singapore

Tel: +65-6321-4677, Fax: +65-6227-3787

E-mail: chokaikyong@gmail.com

ORCID: https://orcid.org/0000-0003-4077-9664

(C) 2021 The Korean Society of Coloproctology

This is an open-access article distributed under the terms of the Creative Commons Attribution NonCommercial License (https://creativecommons.org/licenses/by-nc/4.0) which permits unrestricted noncommercial use, distribution, and reproduction in any medium, provided the original work is properly cited. rence-free survival (RFS) [1]. It has also been often used as a surrogate marker of systemic inflammatory response.

The link between individual inflammatory response and cancer development has been first suggested by Virchow in 1881 [2], and studies in recent years have investigated the utility of inflammatory biomarkers: plasma C-reactive protein, albumin, total white blood cell (TWBC) count and derived differential ratios such as NLR [3], and their prognostic value on various malignancies [4-7] including CRC [8].

CRC patients of $10 \%$ to $20 \%$ first present with bowel obstruction $[9,10]$. While upfront surgery was often indicated, since the introduction of endoscopic self-expandable metallic stent (SEMS) placement for CRC in 1991 [11], the use of SEMS as a bridge to elective curative surgery has been gaining acceptance and increasingly adopted for patients presenting with bowel obstruction [12]. 
Among these patients, the prognostic accuracy of NLR may be influenced by the presence of tumor obstruction [13], obstructive ileocolitis [14], and resultant dehydration. In addition, endoscopic stenting can induce a local desmoplastic reaction and trigger an inflammatory response $[10,15]$. These factors may further confound TWBC and NLR values. Therefore, it remains unclear whether NLR is a useful prognosticating tool in patients with obstructing CRCs who have undergone SEMS placement prior to curative surgery.

This study evaluates the prognostic value of NLR among patients with obstructing CRC who were successfully stented as a bridge to curative surgery.

\section{METHODS}

Between January 2007 and December 2016, 62 patients presented with obstructing CRC at Singapore General Hospital, and underwent endoscopic placement of SEMS as a bridge to curative surgery. Large bowel obstruction was confirmed clinically and radiologically. All patients underwent a computed tomography (CT) scan of the abdomen and pelvis at initial presentation. Full staging CT scans were done either at diagnosis or within 30 days of presentation. There was no evidence of distant metastasis within this cohort of patients. All patients received antibiotics upon admission, before the stenting procedure was commenced. One technical failure and one stent perforation were encountered, with both patients requiring emergency operation on the same day. Three other patients had minimal bowel decompression after endoscopic placement of SEMS and were operated within 48 hours. A total of 57 patients who had successful bowel decompression after SEMS deployment, followed by elective curative surgery, were included in this study. The study was approved by the Institutional Review Board of Singapore General Hospital (No. 2017/2481).

Clinical, histopathological, survival, and oncological data were retrospectively reviewed from a prospectively maintained database. Clinical data including patient demographics, biochemical results, and radiological findings were collected from a computerized database (Sunrise Clinical Manager ver. 5.8, Eclipsys Corp., Atlanta, GA, USA). Endoscopic and surgical data were collected from another server (OTM 10, IBM Corp., Armonk, NY, USA).

NLR was calculated from the differential WBC counts obtained at presentation, and at least 4 days after successful SEMS deployment, before elective surgery. Apical lymph node was defined as the lymph node at the origin of the inferior mesenteric artery. Where possible, all patients were considered for adjuvant chemotherapy in the form of capecitabine and oxaliplatin under the guidance of the oncologist. The postoperative surveillance protocol has been described in an earlier study [16]. RFS was defined as the time period from surgery to the date of detection of radiological and/or pathological CRC recurrence. OS was calculated from the time of surgery to the date of death or latest date of follow-up.

Patient demographics and tumor characteristics were presented in median (interquartile range) for continuous variables and count (percentage) for categorical variables. The OS of 3- and 5 -year and the $95 \%$ confidence interval (CI) were presented. Differences in clinicopathological features between the 2 groups were analyzed using the chi-square or Fisher exact test for categorical variables where appropriate, and the Student $t$-test for continuous variables. Each variable was analyzed by univariate Cox regression model for OS and RFS, respectively. Those variables with Pvalue of $<0.10$ and clinically relevant variables were entered into multivariable Cox regression model. The variables were selected into the respective final model using stepwise approach with significant entry level at 0.10 and significant exit level at 0.05 . The results of Cox regression model were presented in estimated hazard ratio (HR) with 95\% CI and Wald test P-value. Both OS and RFS curves were estimated using Kaplan-Meier method and plotted up to 72 months.

Optimal cutoff to dichotomize NLR was obtained by maximizing log-rank test statistic with recursive partitioning of KaplanMeier RFS and OS curves. Cutoff was initiated from 2.0 with 0.5 increment for subsequent test. Optimal cutoff was determined once 2 greater consecutive P-values of log-rank test were obtained. For NLR values calculated at presentation before stent deployment, the optimal cutoff value was NLR $\geq 5$. As for NLR values obtained after stent deployment before elective curative surgery, the optimal cutoff for high NLR was $\geq 4$ (Supplementary Tables 1 , 2; Supplementary Fig. 1). Statistical analysis was done using SAS ver. 9.4 (SAS Institute, Cary, NC, USA).

\section{RESULTS}

Fifty-seven patients with obstructing CRC were decompressed successfully via SEMS placement, followed by elective curative resection of the primary tumor. There were 23 patients in the high NLR (H-NLR) group with preoperative NLR of $\geq 4$ and 34 patients in the low NLR (L-NLR) group with preoperative NLR of $<4$. The clinical and oncological details of the patients are summarized in Table 1 . The median duration from stenting to surgery of 10.5 days (range, 6 to 23) in the H-NLR group did not differ significantly from the 10 days (range, 5 to 18 ) in the L-NLR group $(\mathrm{P}=0.913)$.

Comparison of baseline characteristics between the 2 groups showed no significant differences in demographics such as age and gender. A majority of the patients were surgically fit (American Society of Anesthesiologists physical status classification I to II) $(n=50)$. In the H-NLR group, there were a greater proportion of patients with elevated preoperative carcinoembryonic antigen (CEA) $5.3 \mu \mathrm{g} / \mathrm{L}$ ( $69.6 \%$ vs. $44.1 \%, \mathrm{P}=0.058$ ), and more patients had T4 tumors (30.4\% vs. $14.7 \%, \mathrm{P}=0.153)$, although these were not statistically significant.

The patients in the H-NLR group were significantly associated with higher rates of apical lymph node involvement, and lymphovascular invasion on histology. The surgical resection margins 
Table 1. Characteristics of 57 patients grouped by neutrophil-to-lymphocyte ratio (NLR)

\begin{tabular}{|c|c|c|c|}
\hline Characteristic & $\mathrm{NLR} \geq 4(\mathrm{n}=23)$ & NLR $<4(n=34)$ & P-value \\
\hline Age (yr) & $74.0(57-81)$ & 71.5 (61.50-78.75) & 0.743 \\
\hline Male sex & $13(56.5)$ & $22(64.7)$ & 0.533 \\
\hline ASA PS classification III & $4(17.4)$ & $3(8.8)$ & 0.423 \\
\hline Diabetes mellitus & $6(26.1)$ & $6(17.6)$ & 0.443 \\
\hline Albumin $(g / L)$ & $36.0(31.0-40.0)$ & $37.0(33.5-40.3)$ & 0.749 \\
\hline CEA $(\mu \mathrm{g} / \mathrm{L})$ & $7.75(4.95-22.20)$ & $4.50(2.55-12.55)$ & 0.412 \\
\hline \multicolumn{4}{|l|}{ Tumor characteristics } \\
\hline Location & & & 0.435 \\
\hline Rectosigmoid & $5(21.7)$ & $3(8.8)$ & \\
\hline Sigmoid & $9(39.1)$ & $15(44.1)$ & \\
\hline Descending & $4(17.4)$ & $12(35.3)$ & \\
\hline Splenic flexure & $5(21.7)$ & $4(11.8)$ & \\
\hline Tumor staging & & & 0.149 \\
\hline T2 & $1(4.3)$ & $0(0)$ & \\
\hline T3 & $15(65.2)$ & $29(85.3)$ & \\
\hline $\mathrm{T} 4$ & $7(30.4)$ & $5(14.7)$ & \\
\hline Nodal involvement & $14(60.9)$ & 18 (52.9) & 0.554 \\
\hline Number of positive nodes & $1(0-3)$ & $1(0-2.25)$ & 0.924 \\
\hline Apical lymph node involved & $5(21.7)$ & $1(2.9)$ & 0.034 \\
\hline Presence of tumor deposit(s) & $3(13.0)$ & $6(17.6)$ & 0.726 \\
\hline Tumor differentiation & & & 0.340 \\
\hline Well-differentiated & $1(4.3)$ & $0(0)$ & \\
\hline Moderately-differentiated & $22(95.7)$ & $33(97.1)$ & \\
\hline Poorly-differentiated & $0(0)$ & $1(2.9)$ & \\
\hline Mucinous component & $1(4.3)$ & $1(2.9)$ & 1.000 \\
\hline Perineural invasion & $10(43.5)$ & $9(26.5)$ & 0.181 \\
\hline Lymphovascular invasion & $12(52.2)$ & $6(17.6)$ & 0.006 \\
\hline Pericolic microabscess & $2(8.7)$ & $5(14.7)$ & 0.689 \\
\hline \multicolumn{4}{|c|}{ Operative and postoperative details } \\
\hline Laparoscopic approach & $10(43.5)$ & $19(55.9)$ & 0.358 \\
\hline Stoma creation & $4(17.4)$ & $0(0)$ & 0.022 \\
\hline Postoperative morbidity & $5(21.7)$ & $5(14.7)$ & 0.493 \\
\hline Adjuvant chemotherapy & $10(43.5)$ & 18 (52.9) & 0.483 \\
\hline Distant metastases & $13(56.5)$ & $11(32.4)$ & 0.070 \\
\hline
\end{tabular}

Values are presented as median (interquartile range) or number (\%).

ASA, American Society of Anesthesiologists; PS, physical status; CEA, carcinoembryonic antigen.

were clear macroscopically and microscopically in all cases within this study, with a mean of 21.7 lymph nodes harvested. While all patients had primary anastomosis after tumor resection, the formation of a temporary diverting stoma was undertaken in 4 patients in the H-NLR group, at the intraoperative discretion of the individual surgeon. Although there were no differences between the 2 groups in terms of the overall rate of postoperative morbidity, 3 patients in the H-NLR group had major complications, including 2 with anastomotic dehiscence and 1 patient who demised postoperatively from septic complications secondary to pneumonia. There was no difference in the proportion of patients who received adjuvant chemotherapy. There was a higher propor- 
tion of H-NLR patients who developed metachronous distant metastases ( $56.5 \%$ vs. $32.4 \%, \mathrm{P}=0.070$ ), with the $\mathrm{P}$-value approaching but not reaching statistical significance.

The median OS for the entire cohort was 70 months (range, 0 to 154 months) (Fig. 1), while the median RFS was 62 months (range, 3 to 154 months). The 5-year OS and RFS were 56\% and $55 \%$, respectively. $\mathrm{H}-\mathrm{NLR}$ of $\geq 4$ was significantly associated with poorer OS and RFS $(\mathrm{P}<0.05)$ on univariate analysis (Figs. 2, 3).

Perineural infiltration, lymphovascular invasion, and absence of adjuvant chemotherapy were associated with worse OS on univariate analysis (Table 2). Major postoperative complication, of Clavien-Dindo grade III and above [17], and NLR of $\geq 4$, negatively impacted OS on both univariate and multivariate analyses.

Among the factors associated with poorer RFS on univariate analysis-T4 tumors, perineural infiltration, lymphovascular in-

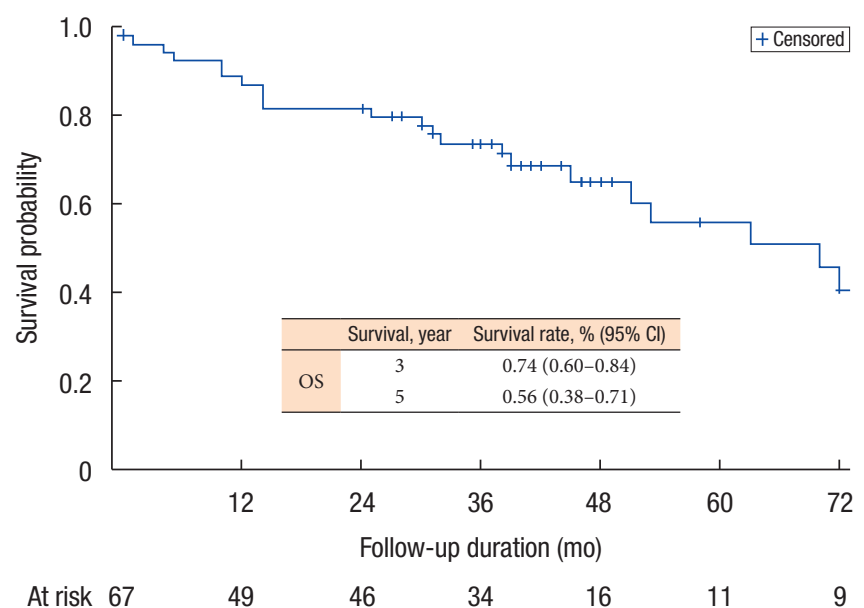

Fig. 1. Overall survival (OS) of the cohort. CI, confidence interval.

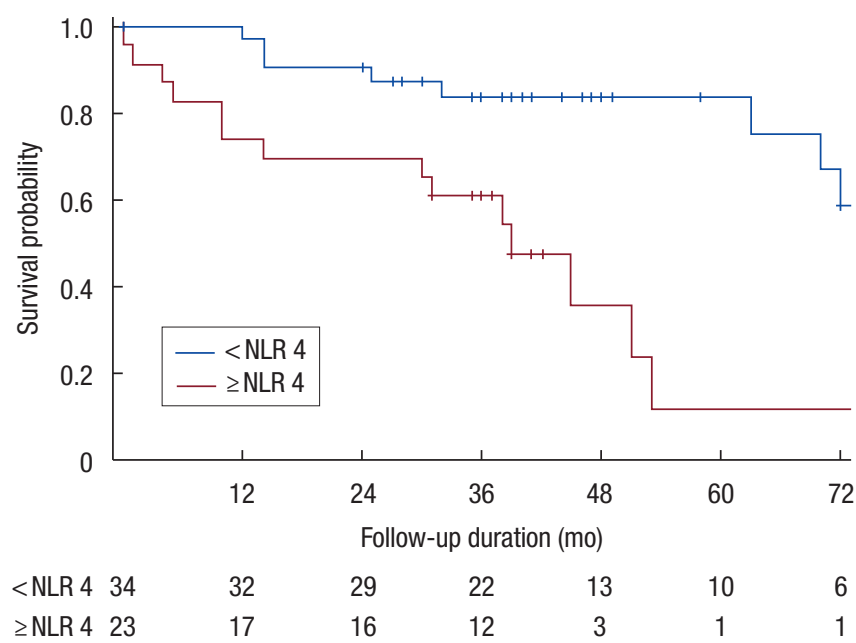

Fig. 2. Overall survival stratified by neutrophil-to-lymphocyte ratio (NLR). vasion, and NLR of $\geq 4$, perineural infiltration was found to be significant on multivariate analysis (Table 3 ).

\section{DISCUSSION}

The role of systemic inflammatory response in predicting oncological outcomes has been well demonstrated. In the presence of ongoing systemic inflammation, there is neutrophilia with a relative lymphocytopenia [18] due to elevation of proinflammatory tumor cells and suppression of cell-mediated immunity [19]. Markers used for inflammatory response include components of TWBC such as NLR, platelet-lymphocyte ratio, and lymphocytemonocyte ratio.

In particular, NLR has been shown to play an important role in predicting the survival of patients with different types of cancers [20], including breast [7], stomach [4], gastrointestinal stromal tumors [5], pancreatic [6] and hepatocellular carcinoma [21]. HNLR has been correlated with cancer invasion, angiogenesis, and metastasis [22]. Indeed, a recent systemic review and meta-analysis by Dolan et al. [23] in 2017 showed that there was a significant association between NLR and OS in patients with operable cancer. The usefulness of NLR in the prognostication of CRC has been widely investigated $[1,8,24-26]$. Nevertheless to the best of our knowledge, the prognostic ability of NLR has not been examined among patients with obstructing CRC who have undergone endoscopic placement of SEMS as a bridge to curative surgery.

In this study, we show that elevated NLR is an independent prognostic factor associated with worse OS on both univariate and multivariate analyses. In addition, elevated NLR is significantly associated with worse RFS on univariate analysis. These findings are consistent with other studies which have reported the association of elevated NLR with adverse oncological outcomes including OS, cancer-specific survival, and RFS, in various co-

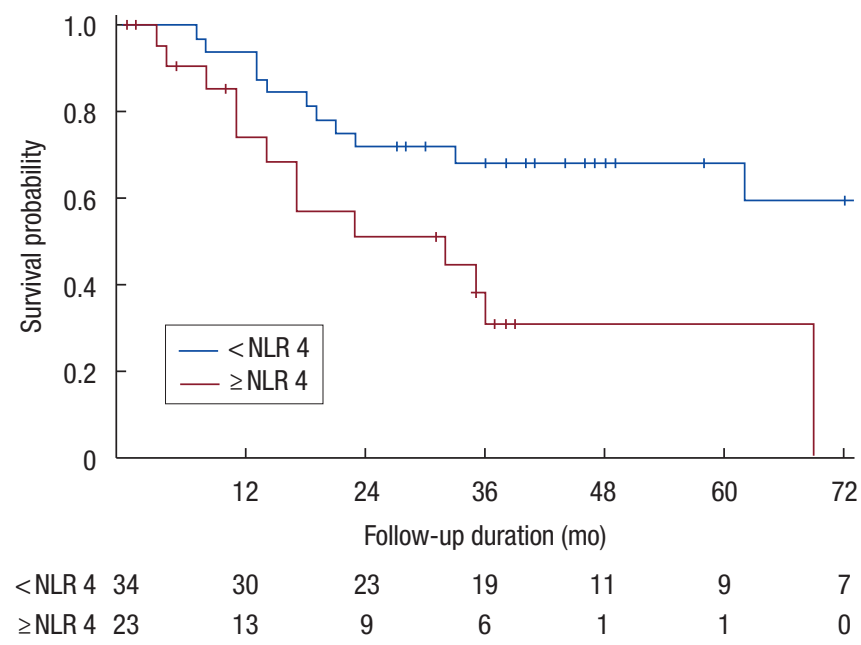

Fig. 3. Recurrence-free survival stratified by neutrophil-to-lymphocyte ratio (NLR). 
Table 2. Prognostic factors influencing overall survival

\begin{tabular}{|c|c|c|c|c|}
\hline \multirow{2}{*}{ Variable } & \multicolumn{2}{|c|}{ Univariate } & \multicolumn{2}{|c|}{ Multivariate } \\
\hline & $\mathrm{HR}(95 \% \mathrm{Cl})$ & P-value & $\mathrm{HR}(95 \% \mathrm{Cl})$ & P-value \\
\hline Age & $1.04(1.00-1.08)$ & 0.088 & $1.04(1.00-1.09)$ & 0.068 \\
\hline ASA PS classification III and above & $2.41(0.88-6.61)$ & 0.087 & & \\
\hline T4 staging & $1.87(0.75-4.61)$ & 0.177 & & \\
\hline Apical LN+ & $2.17(0.62-7.62)$ & 0.227 & & \\
\hline Poor differentiation & $1.56(0.20-11.92)$ & 0.667 & & \\
\hline Tumor deposit(s) & $1.82(0.66-5.02)$ & 0.250 & & \\
\hline Perineural infiltration & $3.05(1.21-7.69)$ & 0.018 & & \\
\hline Lymphovascular invasion & $3.52(1.51-8.21)$ & 0.004 & & \\
\hline Pericolic microabscess & $1.34(0.40-4.52)$ & 0.637 & & \\
\hline Major postoperative complication(s) & $13.55(3.62-50.81)$ & $<0.001$ & $11.34(2.49-51.56)$ & 0.002 \\
\hline Chemotherapy & $0.41(0.17-0.95)$ & 0.039 & & \\
\hline$N L R \geq 4$ & $4.97(2.02-12.21)$ & $<0.001$ & $3.69(1.46-9.35)$ & 0.006 \\
\hline
\end{tabular}

HR, hazard ratio; Cl, confidence interval; ASA, American Society of Anesthesiologists; PS, physical status; LN+, lymph node positive; NLR, neutrophil-to-lymphocyte ratio.

Table 3. Prognostic factors influencing recurrence-free survival

\begin{tabular}{|c|c|c|c|c|}
\hline \multirow{2}{*}{ Variable } & \multicolumn{2}{|c|}{ Univariate analysis } & \multicolumn{2}{|c|}{ Multivariable } \\
\hline & $\mathrm{HR}(95 \% \mathrm{Cl})$ & P-value & $\mathrm{HR}(95 \% \mathrm{Cl})$ & P-value \\
\hline Age & $1.00(0.96-1.03)$ & 0.863 & & \\
\hline ASA PS classification III and above & $1.63(0.48-5.49)$ & 0.432 & & \\
\hline T4 staging & $2.97(1.29-6.60)$ & 0.011 & & \\
\hline Apical LN+ & $2.55(0.75-8.73)$ & 0.136 & & \\
\hline Poor differentiation & $1.46(0.19-11.03)$ & 0.712 & & \\
\hline Tumor deposit(s) & $1.66(0.61-4.47)$ & 0.319 & & \\
\hline Perineural invasion & 4.59 (1.93-10.92) & $<0.001$ & $3.37(1.33-8.55)$ & 0.011 \\
\hline Lymphovascular invasion & $2.97(1.32-6.67)$ & 0.009 & $1.85(0.74-4.62)$ & 0.186 \\
\hline Pericolic microabscess & $0.94(0.22-4.03)$ & 0.939 & & \\
\hline Major postoperative complication(s) & $7.98(0.96-66.28)$ & 0.055 & & \\
\hline Chemotherapy & $1.04(0.46-2.34)$ & 0.921 & & \\
\hline$N L R \geq 4$ & $2.91(1.29-6.60)$ & 0.010 & $1.63(0.63-4.22)$ & 0.312 \\
\hline
\end{tabular}

HR, hazard ratio; Cl, confidence interval; ASA, American Society of Anesthesiologists; PS, physical status; LN+, lymph node positive; NLR, neutrophil-to-lymphocyte ratio.

horts of CRC patients $[8,25,27,28]$.

The NLR value of 4 after stenting was determined to be the most optimal threshold in differentiating survival outcomes in our study. Similarly, from the NLR values obtained at presentation before endoscopic stenting, the optimal value that best-dichotomized outcomes was 5 . Both these NLR cutoff values, obtained before and after stent deployment, are consistent with the NLR threshold of between 3 to 5 reported in most studies examining the prognosticating value of NLR in CRC [23]. The effect of treatment of these obstructed patients with intravenous hydration and antibiotics is likely to account for the lower NLR threshold of 4 after endoscopic stent deployment. Therefore, the applicability of
NLR as a simple and inexpensive biomarker-correlating inflammatory response to oncological outcomes $[27,29]$, can be extended to this subset of patients with obstructing localized CRC who are first decompressed by endoscopic stent placement followed by elective curative surgery.

Based on our cutoff value of 4 , we found that elevated NLR after endoscopic stent placement was significantly associated with higher rates of apical lymph node involvement and lymphovascular invasion-pathologic indicators of adverse oncological outcome. Within the H-NLR group, the median CEA level was higher (7.75 vs. $4.50 \mu \mathrm{g} / \mathrm{L})$ and there was a larger proportion of $\mathrm{T} 4$ tumors (30.4\% vs. $14.7 \%)$, although these did not approach statis- 
tical significance. The 5-year OS and RFS of 56\% and 55\% respectively in our cohort. Similarly, a recent systemic review by Cao et al. [30] reported the 5-year OS ranging between 57\% and 63\% among patients with obstructing CRC.

The overall proportion of patients who completed adjuvant chemotherapy was $49.1 \%$ in this study, including 15 patients who declined further chemotherapy treatment postoperatively, whereas the remaining patients were unable to complete the full regimen due to side effects. While the proportion of patients in the H-NLR group vs. L-NLR group receiving chemotherapy were similar, there was a slightly higher rate of metachronous distant metastases observed among patients in the H-NLR group. With today's emergence of more individualized treatment strategies, the oncologic prognostic significance of NLR can potentially aid the selection of our patients for adjuvant treatment or intensification of postoperative surveillance, in addition to the clinicopathological factors currently used.

The main limitations of this study are its retrospective nature and relatively small cohort size. Nonetheless, we demonstrate that the prognostic ability of NLR remains relevant among patients with obstructing localized CRC who are decompressed by endoscopic stent placement as a bridge to elective curative surgery.

In conclusion, NLR of $\geq 4$ after stenting is an independent prognostic factor associated with worse OS and RFS, among patients with obstructing localized CRC who are decompressed by endoscopic stent placement as a bridge to elective curative surgery. NLR is a useful predictor of more advanced tumor biology, associated with higher rates of apical lymph node involvement and lymphovascular invasion.

\section{CONFLICT OF INTEREST}

No potential conflict of interest relevant to this article was reported.

\section{SUPPLEMENTARY MATERIALS}

Supplementary materials for this study are presented online (available at https://doi.org/10.3393/ac.2020.05.25).

\section{REFERENCES}

1. Haram A, Boland MR, Kelly ME, Bolger JC, Waldron RM, Kerin MJ. The prognostic value of neutrophil-to-lymphocyte ratio in colorectal cancer: a systematic review. J Surg Oncol 2017;115:470-9.

2. Virchow R. An address on the value of pathological experiments. Br Med J 1881;2:198-203.

3. Paik KY, Lee IK, Lee YS, Sung NY, Kwon TS. Clinical implications of systemic inflammatory response markers as independent prognostic factors in colorectal cancer patients. Cancer Res Treat 2014; 46:65-73.

4. Miyamoto R, Inagawa S, Sano N, Tadano S, Adachi S, Yamamoto
M. The neutrophil-to-lymphocyte ratio (NLR) predicts shortterm and long-term outcomes in gastric cancer patients. Eur J Surg Oncol 2018;44:607-12.

5. Goh BK, Chok AY, Allen JC Jr, Quek R, Teo MC, Chow PK, et al. Blood neutrophil-to-lymphocyte and platelet-to-lymphocyte ratios are independent prognostic factors for surgically resected gastrointestinal stromal tumors. Surgery 2016;159:1146-56.

6. Stotz M, Gerger A, Eisner F, Szkandera J, Loibner H, Ress AL, et al. Increased neutrophil-lymphocyte ratio is a poor prognostic factor in patients with primary operable and inoperable pancreatic cancer. Br J Cancer 2013;109:416-21.

7. Duan J, Pan L, Yang M. Preoperative elevated neutrophil-to-lymphocyte ratio (NLR) and derived NLR are associated with poor prognosis in patients with breast cancer: a meta-analysis. Medicine (Baltimore) 2018;97:e13340.

8. Walsh SR, Cook EJ, Goulder F, Justin TA, Keeling NJ. Neutrophillymphocyte ratio as a prognostic factor in colorectal cancer. J Surg Oncol 2005;91:181-4.

9. Phillips RK, Hittinger R, Fry JS, Fielding LP. Malignant large bowel obstruction. Br J Surg 1985;72:296-302.

10. Kim EJ, Kim YJ. Stents for colorectal obstruction: past, present, and future. World J Gastroenterol 2016;22:842-52.

11. Dohmoto M. New method-endoscopic implantation of rectal stent in palliative treatment of malignant stenosis. Endosc Dig 1991;3:1507-12.

12. Foo CC, Poon SH, Chiu RH, Lam WY, Cheung LC, Law WL. Is bridge to surgery stenting a safe alternative to emergency surgery in malignant colonic obstruction: a meta-analysis of randomized control trials. Surg Endosc 2019;33:293-302.

13. Maruthachalam K, Lash GE, Shenton BK, Horgan AF. Tumour cell dissemination following endoscopic stent insertion. $\mathrm{Br} J$ Surg 2007;94:1151-4.

14. Pang NQ, Lim TZ, Zhou Y, Tan KK. Obstructive ileocolitis in patients with obstructed colorectal cancer: a matched case control study. Ann Coloproctol 2018;34:175-9.

15. Coussens LM, Werb Z. Inflammation and cancer. Nature 2002; 420:860-7.

16. Chok AY, Lim HJ, Lye WK, Samarakoon LB, Guo J, Tang CL, et al. Stenting as a bridge to surgery for obstructed stage IV colorectal cancers: long-term outcomes of a 10-year study. Asian J Endosc Surg 2020;13:343-50.

17. Dindo D, Demartines N, Clavien PA. Classification of surgical complications: a new proposal with evaluation in a cohort of 6336 patients and results of a survey. Ann Surg 2004;240:205-13.

18. Zahorec R. Ratio of neutrophil to lymphocyte counts: rapid and simple parameter of systemic inflammation and stress in critically ill. Bratisl Lek Listy 2001;102:5-14.

19. Grivennikov SI, Greten FR, Karin M. Immunity, inflammation, and cancer. Cell 2010;140:883-99.

20. Roxburgh CS, McMillan DC. Role of systemic inflammatory response in predicting survival in patients with primary operable cancer. Future Oncol 2010;6:149-63. 
21. Najjar M, Agrawal S, Emond JC, Halazun KJ. Pretreatment neutrophil-lymphocyte ratio: useful prognostic biomarker in hepatocellular carcinoma. J Hepatocell Carcinoma 2018;5:17-28.

22. Kitamura T, Qian BZ, Pollard JW. Immune cell promotion of metastasis. Nat Rev Immunol 2015;15:73-86.

23. Dolan RD, Lim J, McSorley ST, Horgan PG, McMillan DC. The role of the systemic inflammatory response in predicting outcomes in patients with operable cancer: systematic review and meta-analysis. Sci Rep 2017;7:16717.

24. Kim H, Jung HI, Kwon SH, Bae SH, Kim HC, Baek MJ, et al. Preoperative neutrophil-lymphocyte ratio and CEA is associated with poor prognosis in patients with synchronous colorectal cancer liver metastasis. Ann Surg Treat Res 2019;96:191-200.

25. Ding PR, An X, Zhang RX, Fang YJ, Li LR, Chen G, et al. Elevated preoperative neutrophil to lymphocyte ratio predicts risk of recurrence following curative resection for stage IIA colon cancer. Int J Colorectal Dis 2010;25:1427-33.

26. Kishi Y, Kopetz S, Chun YS, Palavecino M, Abdalla EK, Vauthey JN. Blood neutrophil-to-lymphocyte ratio predicts survival in patients with colorectal liver metastases treated with systemic che- motherapy. Ann Surg Oncol 2009;16:614-22.

27. Hung HY, Chen JS, Yeh CY, Changchien CR, Tang R, Hsieh PS, et al. Effect of preoperative neutrophil-lymphocyte ratio on the surgical outcomes of stage II colon cancer patients who do not receive adjuvant chemotherapy. Int J Colorectal Dis 2011;26:105965.

28. Jankova L, Dent OF, Chan C, Chapuis P, Clarke SJ. Preoperative neutrophil/lymphocyte ratio predicts overall survival but does not predict recurrence or cancer-specific survival after curative resection of node-positive colorectal cancer. BMC Cancer 2013;13:442.

29. Shibutani M, Maeda K, Nagahara H, Noda E, Ohtani H, Nishiguchi Y, et al. A high preoperative neutrophil-to-lymphocyte ratio is associated with poor survival in patients with colorectal cancer. Anticancer Res 2013;33:3291-4.

30. Cao Y, Gu J, Deng S, Li J, Wu K, Cai K. Long-term tumour outcomes of self-expanding metal stents as 'bridge to surgery' for the treatment of colorectal cancer with malignant obstruction: a systematic review and meta-analysis. Int J Colorectal Dis 2019;34: 1827-38. 